\title{
Gastric Intestinal Metaplasia and Tamoxifen: Can We Reverse the Inevitable?
}

\author{
James R. Goldenring
}

Published online: 5 March 2014

(c) Springer Science+Business Media New York 2014

Gastric intestinal metaplasia can occur after chronic infection with Helicobacter pylori and is considered a precursor of gastric adenocarcinoma. Thus, interventions which can reverse intestinal metaplasia may reduce the incidence of gastric cancer. The manuscript by Moon et al. published in this issue of Digestive Diseases and Sciences [1] reports somewhat surprising results suggesting that Korean women receiving chronic tamoxifen treatment after breast cancer surgery showed a decrease in intestinal metaplasia. Previous work from the Mills laboratory had shown that high doses of tamoxifen treatment in mice led to loss of parietal cells and the evolution of Spasmolytic Polypeptide Expressing Metaplasia (SPEM). Nevertheless, in patients receiving lower therapeutic doses of tamoxifen for an extended period, the findings of Moon et al. indicate that tamoxifen may in fact impair progression of metaplasia to intestinal metaplasia. Importantly, since patients taking aromatase inhibitors did not alter the extent of intestinal metaplasia, the tamoxifen effects observed are likely due to an off-target effect rather than one associated with estrogen receptor blockade. Previous investigations have noted off-target effects of tamoxifen at various levels including alterations in calcium signaling [2], ceramide metabolism [3] and the parietal cell protonophore activities previously proposed by the Mills laboratory [4]. Thus, while it is difficult to define the mechanisms that may be responsible for the observed mucosal alterations in this treatment cohort, the results are nevertheless encouraging

J. R. Goldenring $(\bowtie)$

Epithelial Biology Center and Section of Surgical Science,

Vanderbilt University Medical Center, 10435-G MRB IV, 2213

Garland Avenue, Nashville, TN 37232, USA

e-mail: jim.goldenring@vanderbilt.edu for the development of interventions which alter the natural history of metaplasia in the stomach.

The authors acknowledge the limitations of this retrospective study. A critical question left by these studies focuses on the impact of tamoxifen treatment on the mucosal lineages. While the report demonstrates that women receiving tamoxifen have a reduced amount of intestinal metaplasia, it is not at all clear that the loss of intestinal metaplasia leads to re-establishment of a normal mucosa. Recent investigations have supported the concept that loss of parietal cells initiated by $H$. pylori infection leads initially to the development of Spasmolytic Polypeptide Expressing Metaplasia (SPEM) and then over time SPEM evolves into intestinal metaplasia [5]. Unfortunately, the present study did not examine either the state of remaining atrophy or the presence of SPEM in tamoxifentreated patients. Interestingly, the progression of metaplasia from SPEM to intestinal metaplasia and neoplasia may be driven by inflammatory cells, especially macrophages [6]. Indeed, alterations in inflammatory cell response are the most likely explanation for the off-target influences of tamoxifen. While the data in the present study suggest that the inflammation scores were not significantly improved by tamoxifen treatment, there is no way to judge whether this qualitative determination would reflect changes in macrophage subsets. Further studies will need to address possible effects of tamoxifen treatment on inflammatory cell function.

Since intestinal metaplasia is considered the critical precursor lesion for the development of gastric adenocarcinoma, the reversibility of metaplastic lesions is of considerable interest. Some investigations have suggested eradication of $H$. pylori may ameliorate or even reverse intestinal metaplasia, but other studies have found significant numbers of patients who retain intestinal metaplasia 
even after effective eradication [7-10]. In identifying putative treatments that might lead to reversal of metaplasia, either through reduction in inflammation or other direct effects, it will be important to perform well-controlled prospective trials with, if possible, correlative preclinical correlative studies. Thus, it is of interest that Saya et al. [11] have recently reported that in GAN mice, which develop spontaneous proximal gastric neoplasms, sulfasalazine treatment ameliorates the development of dysplastic lesions, with treated mice showing a relatively benign pathology with SPEM and foveolar hyperplasia. In human studies, future evaluation of any agents targeting intestinal metaplasia must assess not only the alterations in intestinal metaplasia, but also the presence of continued parietal cell loss (oxyntic atrophy), the presence of SPEM and the effects of treatment on the character of immune cell infiltrates. Given the range of deleterious side effects that accrue from chronic tamoxifen treatment, it seems unlikely that this drug will find use in the treatment of patients with intestinal metaplasia. Nevertheless, the findings of Moon, et al. do suggest that discrete pharmacological interventions may alter the course of metaplastic progression. Less toxic therapeutics, like sulfasalazine, may find utility in the future for treatment of patients with intestinal metaplasia, who are at increased risk for developing gastric cancer.

\section{References}

1. Moon CM, Kim SH, Lee SK et al. Chronic tamoxifen use is associated with a decreased risk of intestinal metaplasia in human gastric epithelium. Dig Dis Sci. (Epub ahead of print). doi:10. 1007/s10620-013-2994-1.

2. Parsons AB, Lopez A, Givoni IE, et al. Exploring the mode-ofaction of bioactive compounds by chemical-genetic profiling in yeast. Cell. 2006;126:611-625.

3. Morad SA, Levin JC, Tan SF, et al. Novel off-target effect of tamoxifen-inhibition of acid ceramidase activity in cancer cells. Biochim Biophys Acta. 2013;1831:1657-1664.

4. Huh WJ, Khurana SS, Geahlen JH, et al. Tamoxifen induces rapid, reversible atrophy, and metaplasia in mouse stomach. Gastroenterology. 2012;142:21-24.

5. Goldenring JR, Nam KT, Wang TC, et al. Spasmolytic polypeptide-expressing metaplasia and intestinal metaplasia: time for reevaluation of metaplasias and the origins of gastric cancer. Gastroenterology. 2010;138(2207-10):2210.

6. Oshima H, Hioki K, Popivanova BK, et al. Prostaglandin E signaling and bacterial infection recruit tumor-promoting macrophages to mouse gastric tumors. Gastroenterology. 2011;140:596-607.

7. Lee YC, Chen TH, Chiu HM, et al. The benefit of mass eradication of Helicobacter pylori infection: a community-based study of gastric cancer prevention. Gut. 2013;62:672-682.

8. Kang JM, Kim N, Shin CM, et al. Predictive factors for improvement of atrophic gastritis and intestinal metaplasia after Helicobacter pylori eradication: a three-year follow-up study in Korea. Helicobacter. 2012;17:86-95.

9. Yoon H, Kim N, Lee HS, et al. Helicobacter pylori-negative gastric cancer in South Korea: incidence and clinicopathologic characteristics. Helicobacter. 2011;16:382-388.

10. de Vries AC, Kuipers EJ, Rauws EA. Helicobacter pylori eradication and gastric cancer: when is the horse out of the barn? Am J Gastroenterol. 2009;104:1342-1345.

11. Wada $\mathrm{T}$, Ishimoto $\mathrm{T}$, Seishima $\mathrm{R}$, et al. Functional role of $\mathrm{CD} 44 \mathrm{v}-\mathrm{xCT}$ system in the development of spasmolytic polypeptide-expressing metaplasia. Cancer Sci. 2013;104:1323-1329. 